Acta Crystallographica Section E

Structure Reports

Online

ISSN 1600-5368

\section{$N$-[3-(2-Methylphenyl)isoquinolin-1-yl]- formamide}

\section{Fu Na Cui, Jun Qi Li, Xiao Li Chen and Qing Bao Song*}

The State Key Laboratory Breeding Base of Green Chemistry-Synthesis Technology, College of Chemical Engineering and Materials Science, Zhejiang University of Technology, Hangzhou 310014, People's Republic of China

Correspondence e-mail: qbsong@zjut.edu.cn

Received 21 March 2009; accepted 30 March 2009

Key indicators: single-crystal X-ray study; $T=296 \mathrm{~K}$; mean $\sigma(\mathrm{C}-\mathrm{C})=0.003 \AA$; $R$ factor $=0.043 ; w R$ factor $=0.124 ;$ data-to-parameter ratio $=13.2$.

The title compound, $\mathrm{C}_{17} \mathrm{H}_{14} \mathrm{~N}_{2} \mathrm{O}$, crystallizes as a cis formamide isomer. The isoquinoline and benzene fragments are nearly perpendicular [dihedral angle $=81.79(18)^{\circ}$ ], whereas the formamide group is virtually coplanar with the isoquinoline unit [dihedral angle $=1.66(15)^{\circ}$ ]. Intermolecular $\mathrm{N}-\mathrm{H}$. . O hydrogen bonds link molecules into a centrosymmetric dimer.

\section{Related literature}

For the cytotoxic activity of arylisoquinolines, see: Cho et al. (2002, 2003). For the synthethic procedures relevant to this work, see: Nunno et al. (2008); Tovar \& Swager (1999); Cho et al. (2002).<smiles>Cc1ccccc1-c1cc2ccccc2c(NC=O)n1</smiles>

\section{Experimental}

Crystal data

$\mathrm{C}_{17} \mathrm{H}_{14} \mathrm{~N}_{2} \mathrm{O}$

$M_{r}=262.30$
Triclinic, $P \overline{1}$

$a=5.3898(14) \AA$

$b=11.166(3) \AA$

$c=11.899(3) \AA$

$\alpha=106.139$ (3)

$\beta=93.128(3)^{\circ}$

$\gamma=103.800(3)^{\circ}$

$V=662.4(3) \AA^{3}$

$Z=2$

Mo $K \alpha$ radiation

$\mu=0.08 \mathrm{~mm}^{-1}$

$T=296 \mathrm{~K}$

$0.36 \times 0.23 \times 0.16 \mathrm{~mm}$

\section{Data collection}

Bruker SMART CCD area-detector diffractometer

Absorption correction: multi-scan (SADABS; Bruker, 2000)

$T_{\min }=0.971, T_{\max }=0.987$

Refinement

$R\left[F^{2}>2 \sigma\left(F^{2}\right)\right]=0.043$

$w R\left(F^{2}\right)=0.124$

$S=1.03$

2399 reflections

4772 measured reflections 2399 independent reflections 1575 reflections with $I>2 \sigma(I)$ $R_{\text {int }}=0.017$

\section{Table 1}

Hydrogen-bond geometry $\left(\AA,^{\circ}\right)$.

\begin{tabular}{lllll}
\hline$D-\mathrm{H} \cdots A$ & $D-\mathrm{H}$ & $\mathrm{H} \cdots A$ & $D \cdots A$ & $D-\mathrm{H} \cdots A$ \\
\hline $\mathrm{N} 2-\mathrm{H} 2 \cdots \mathrm{O} 1^{\mathrm{i}}$ & 0.86 & 2.10 & $2.940(2)$ & 165 \\
\hline
\end{tabular}

Symmetry code: (i) $-x+1,-y+1,-z+1$.

Data collection: SMART (Bruker, 2000); cell refinement: SAINT (Bruker, 2000); data reduction: SAINT; program(s) used to solve structure: SHELXS97 (Sheldrick, 2008); program(s) used to refine structure: SHELXL97 (Sheldrick, 2008); molecular graphics: SHELXTL (Sheldrick, 2008); software used to prepare material for publication: SHELXTL.

This work was supported by the Key Discipline of Applied Chemistry, Zhejiang Province, and the State Key Laboratory Breeding Base of Green Chemistry-Synthesis Technology, Zhejiang University of Technology, People's Republic of China.

Supplementary data and figures for this paper are available from the IUCr electronic archives (Reference: GK2201).

\section{References}

Bruker (2000). SMART, SAINT and SADABS. Bruker AXS Inc., Madison, Wisconsin, USA.

Cho, W. J., Kim, E. K., Park, Y., Jeong, E. Y., Kim, T. S., Le, T. N., Kim, D. D. \& Lee, E. S. (2002). Bioorg. Med. Chem. 10, 2953-2961.

Cho, W. J., Min, S. Y., Le, T. N. \& Kim, T. S. (2003). Bioorg. Med. Chem. Lett. 13, 4451-4454.

Nunno, L. D., Vitale, P. \& Scilimati, A. (2008). Tetrahedron, 64, 11198-11204. Sheldrick, G. M. (2008). Acta Cryst. A64, 112-122.

Tovar, J. D. \& Swager, T. M. (1999). J. Org. Chem. 64, 6499-6505. 


\section{supporting information}

Acta Cryst. (2009). E65, o960 [doi:10.1107/S1600536809011714]

\section{N-[3-(2-Methylphenyl)isoquinolin-1-yl] formamide}

\section{Fu Na Cui, Jun Qi Li, Xiao Li Chen and Qing Bao Song}

\section{S1. Comment}

Many of the arylisoquinoline derivatives exhibit potent cytotoxic activities against five different human tumor cell lines (Cho et al., 2002, 2003). The title compound, that belongs to arylisoquinolines, has been synthesized to study its cytotoxic activity and its crystal structure is reported here.

\section{S2. Experimental}

A 2.5 $\mathrm{M}$ solution of $\mathrm{n}-\mathrm{BuLi}$ in hexanes $(54.5 \mathrm{mmol})$ was added to a solution of the diisopropylamine $(59.9 \mathrm{mmol})$ in THF $(5 \mathrm{ml})$ at $273 \mathrm{~K}$ under nitrogen atmosphere. After $10 \mathrm{~min}$, the solution of 2-methylbenzonitrile (36.4 mmol) in THF (5 ml) was added dropwise and the obtained brown reaction mixture was stirred for $1 \mathrm{~h}$, then adding the DMF (18.2 mmol), the mixture was stirred for $2 \mathrm{~h}$ at room temperature (Cho et al., 2002; Nunno et al., 2008; Tovar et al., 1999). The mixture was subsequently concentrated under reduced pressure giving the crude product. The residue was recrystallized from ethanol. Colorless crystals of the title compound were obtained by slow evaporation of the solvent after 2 days at room temperature(Yield: 73\%, m.p. 401-403 K).

\section{S3. Refinement}

All $\mathrm{H}$ atoms were placed in calculated posistion with $\mathrm{C}-\mathrm{H}=0.93-0.96 \AA$, and $\mathrm{N}-\mathrm{H}=0.86 \AA$ and refined in the riding mode aproximation with $U_{\text {iso }}(\mathrm{H})=1.2 U_{\text {eq }}$ of the carrier atom. 


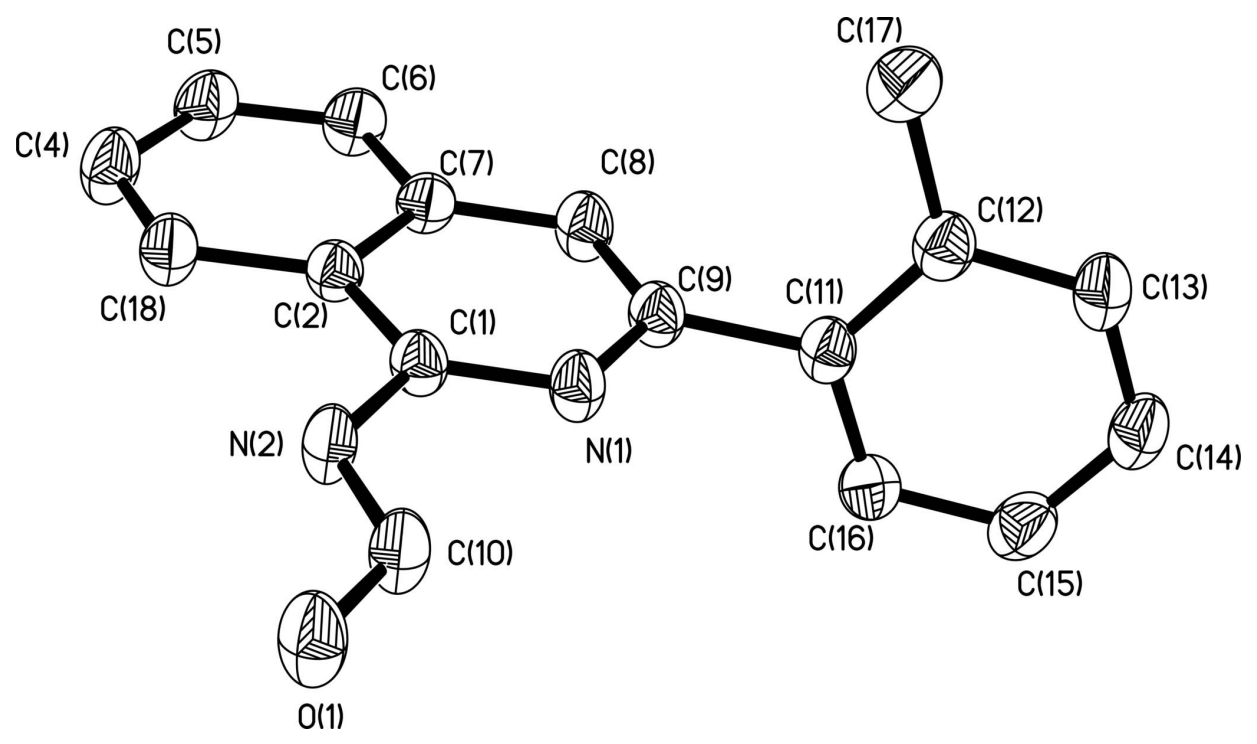

\section{Figure 1}

View of the molecular structure showing the atom-labelling scheme. Displacement ellipsoids are drawn at the 40\% probability level. $\mathrm{H}$ atoms have been omitted for clarity.

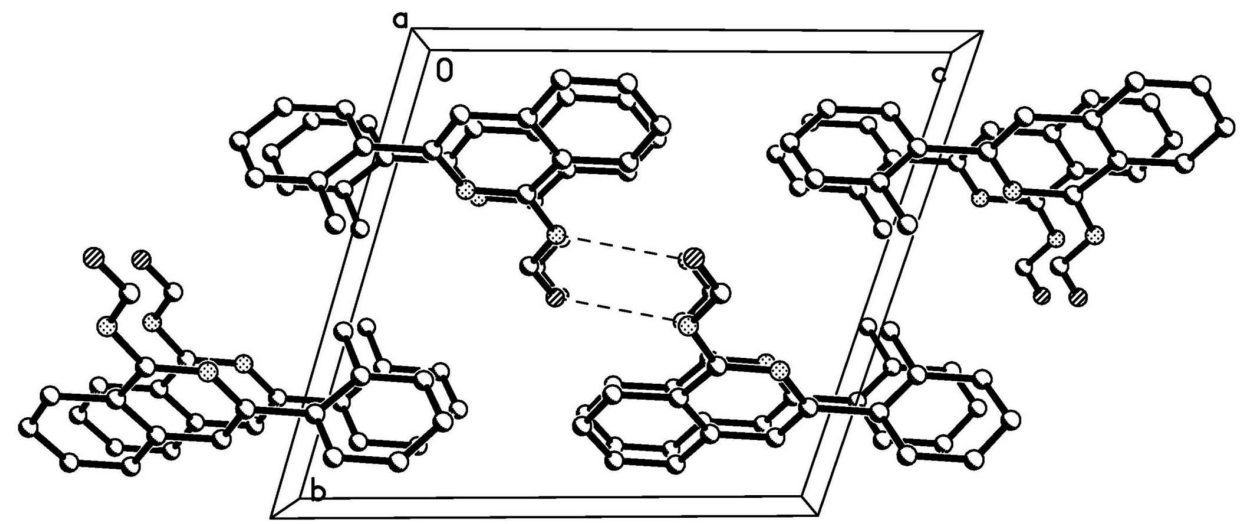

\section{Figure 2}

The molecular packing viewed along the $a$ axis. Hydrogen bonds are shown with dashed lines. $\mathrm{H}$ atoms are omitted for clarity.

\section{$N$-[3-(2-Methylphenyl)isoquinolin-1-yl]formamide}

\section{Crystal data}

$\mathrm{C}_{17} \mathrm{H}_{14} \mathrm{~N}_{2} \mathrm{O}$

$M_{r}=262.30$

Triclinic, $P \overline{1}$

Hall symbol: -P 1

$a=5.3898(14) \AA$

$b=11.166(3) \AA$

$c=11.899(3) \AA$

$\alpha=106.139(3)^{\circ}$

$\beta=93.128(3)^{\circ}$

$\gamma=103.800(3)^{\circ}$

$V=662.4(3) \AA^{3}$
$Z=2$

$F(000)=276$

$D_{\mathrm{x}}=1.315 \mathrm{Mg} \mathrm{m}^{-3}$

Mo $K \alpha$ radiation, $\lambda=0.71073 \AA$

Cell parameters from 1222 reflections

$\theta=3.0-26.0^{\circ}$

$\mu=0.08 \mathrm{~mm}^{-1}$

$T=296 \mathrm{~K}$

Block, colourless

$0.36 \times 0.23 \times 0.16 \mathrm{~mm}$ 


\section{Data collection}

Bruker SMART CCD area-detector diffractometer

Radiation source: fine-focus sealed tube Graphite monochromator

Detector resolution: 0 pixels $\mathrm{mm}^{-1}$

$\varphi$ and $\omega$ scans

Absorption correction: multi-scan (SADABS; Bruker, 2000)

$T_{\min }=0.971, T_{\max }=0.987$

Refinement

Refinement on $F^{2}$

Least-squares matrix: full

$R\left[F^{2}>2 \sigma\left(F^{2}\right)\right]=0.043$

$w R\left(F^{2}\right)=0.124$

$S=1.03$

2399 reflections

182 parameters

0 restraints

Primary atom site location: structure-invariant direct methods
4772 measured reflections

2399 independent reflections

1575 reflections with $I>2 \sigma(I)$

$R_{\text {int }}=0.017$

$\theta_{\max }=25.5^{\circ}, \theta_{\min }=3.0^{\circ}$

$h=-6 \rightarrow 6$

$k=-13 \rightarrow 13$

$l=-14 \rightarrow 14$

Secondary atom site location: difference Fourier map

Hydrogen site location: inferred from neighbouring sites

$\mathrm{H}$-atom parameters constrained

$w=1 /\left[\sigma^{2}\left(F_{\mathrm{o}}^{2}\right)+(0.0578 P)^{2}+0.0805 P\right]$

where $P=\left(F_{\mathrm{o}}{ }^{2}+2 F_{\mathrm{c}}{ }^{2}\right) / 3$

$(\Delta / \sigma)_{\max }<0.001$

$\Delta \rho_{\max }=0.13 \mathrm{e} \AA^{-3}$

$\Delta \rho_{\min }=-0.19 \mathrm{e} \AA^{-3}$

Special details

Geometry. All e.s.d.'s (except the e.s.d. in the dihedral angle between two 1.s. planes)

are estimated using the full covariance matrix. The cell e.s.d.'s are taken

into account individually in the estimation of e.s.d.'s in distances, angles

and torsion angles; correlations between e.s.d.'s in cell parameters are only

used when they are defined by crystal symmetry. An approximate (isotropic)

treatment of cell e.s.d.'s is used for estimating e.s.d.'s involving 1.s. planes.

Refinement. Refinement of $F^{2}$ against ALL reflections. The weighted $R$-factor $w R$ and goodness of fit $S$ are based on $F^{2}$, conventional $R$-factors $R$ are based on $F$, with $F$ set to zero for negative $F^{2}$. The threshold expression of $F^{2}>\sigma\left(F^{2}\right)$ is used only for calculating $R$-factors(gt) $e t c$. and is not relevant to the choice of reflections for refinement. $R$-factors based on $F^{2}$ are statistically about twice as large as those based on $F$, and $R$ - factors based on ALL data will be even larger.

Fractional atomic coordinates and isotropic or equivalent isotropic displacement parameters $\left(\hat{A}^{2}\right)$

\begin{tabular}{lllll}
\hline & $x$ & $y$ & $z$ & $U_{\text {iso }} * / U_{\text {eq }}$ \\
\hline C1 & $0.2507(3)$ & $0.67650(17)$ & $0.71903(14)$ & $0.0446(4)$ \\
C2 & $0.1010(3)$ & $0.74496(17)$ & $0.67117(15)$ & $0.0444(4)$ \\
C4 & $-0.0799(4)$ & $0.8081(2)$ & $0.51425(18)$ & $0.0651(6)$ \\
H4 & -0.1015 & 0.8032 & 0.4349 & $0.078^{*}$ \\
C5 & $-0.1976(4)$ & $0.8866(2)$ & $0.59439(18)$ & $0.0627(6)$ \\
H5 & -0.2959 & 0.9339 & 0.5681 & $0.075^{*}$ \\
C6 & $-0.1697(4)$ & $0.8945(2)$ & $0.71022(18)$ & $0.0592(5)$ \\
H6 & -0.2504 & 0.9465 & 0.7627 & $0.071^{*}$ \\
C7 & $-0.0188(3)$ & $0.82420(18)$ & $0.75214(15)$ & $0.0481(5)$ \\
C8 & $0.0201(4)$ & $0.83123(19)$ & $0.87248(16)$ & $0.0550(5)$ \\
H8 & -0.0586 & 0.8820 & 0.9272 & $0.066^{*}$ \\
C9 & $0.1717(4)$ & $0.76428(18)$ & $0.90878(15)$ & $0.0485(5)$ \\
C10 & $0.5217(4)$ & $0.52789(19)$ & $0.67853(16)$ & $0.0551(5)$ \\
H10 & 0.5431 & 0.5346 & 0.7583 & $0.066^{*}$
\end{tabular}




$\begin{array}{lllll}\text { C11 } & 0.2307(4) & 0.77304(19) & 1.03607(15) & 0.0479(5) \\ \text { C12 } & 0.0620(4) & 0.69894(19) & 1.09041(16) & 0.0531(5) \\ \text { C13 } & 0.1360(4) & 0.7069(2) & 1.20679(17) & 0.0623(6) \\ \text { H13 } & 0.0261 & 0.6567 & 1.2437 & 0.075^{*} \\ \text { C14 } & 0.3655(4) & 0.7863(2) & 1.26835(18) & 0.0636(6) \\ \text { H14 } & 0.4105 & 0.7889 & 1.3457 & 0.076^{*} \\ \text { C15 } & 0.5290(4) & 0.8620(2) & 1.21612(18) & 0.0662(6) \\ \text { H15 } & 0.6831 & 0.9178 & 1.2584 & 0.079^{*} \\ \text { C16 } & 0.4630(4) & 0.8548(2) & 1.09957(17) & 0.0600(6) \\ \text { H16 } & 0.5751 & 0.9051 & 1.0635 & 0.072^{*} \\ \text { C17 } & -0.1907(4) & 0.6097(2) & 1.0258(2) & 0.0735(6) \\ \text { H17A } & -0.3010 & 0.6596 & 1.0076 & 0.110^{*} \\ \text { H17B } & -0.2710 & 0.5602 & 1.0746 & 0.110^{*} \\ \text { H17C } & -0.1617 & 0.5524 & 0.9541 & 0.110^{*} \\ \text { C18 } & 0.0666(4) & 0.7385(2) & 0.55094(16) & 0.0558(5) \\ \text { H18 } & 0.1441 & 0.6866 & 0.4966 & 0.067^{*} \\ \text { N1 } & 0.2872(3) & 0.68582(15) & 0.83158(12) & 0.0492(4) \\ \text { N2 } & 0.3716(3) & 0.59354(15) & 0.64362(12) & 0.0517(4) \\ \text { H2 } & 0.3469 & 0.5844 & 0.5694 & 0.062^{*} \\ \text { O1 } & 0.6325(3) & 0.45974(14) & 0.61283(11) & 0.0655(4) \\ & & & & \end{array}$

Atomic displacement parameters $\left(\AA^{2}\right)$

\begin{tabular}{lllllll}
\hline & $U^{11}$ & $U^{22}$ & $U^{33}$ & $U^{12}$ & $U^{13}$ & $U^{23}$ \\
\hline C1 & $0.0521(11)$ & $0.0470(11)$ & $0.0386(10)$ & $0.0214(9)$ & $0.0112(8)$ & $0.0112(8)$ \\
C2 & $0.0477(10)$ & $0.0478(11)$ & $0.0410(10)$ & $0.0187(9)$ & $0.0078(8)$ & $0.0134(8)$ \\
C4 & $0.0777(15)$ & $0.0831(16)$ & $0.0477(11)$ & $0.0425(13)$ & $0.0049(10)$ & $0.0231(11)$ \\
C5 & $0.0683(13)$ & $0.0731(15)$ & $0.0596(12)$ & $0.0387(12)$ & $0.0033(10)$ & $0.0241(11)$ \\
C6 & $0.0639(13)$ & $0.0663(14)$ & $0.0569(12)$ & $0.0364(11)$ & $0.0094(10)$ & $0.0168(10)$ \\
C7 & $0.0492(11)$ & $0.0519(12)$ & $0.0466(10)$ & $0.0206(9)$ & $0.0068(8)$ & $0.0142(9)$ \\
C8 & $0.0668(13)$ & $0.0630(13)$ & $0.0426(10)$ & $0.0340(11)$ & $0.0147(9)$ & $0.0119(9)$ \\
C9 & $0.0555(11)$ & $0.0555(12)$ & $0.0384(10)$ & $0.0238(10)$ & $0.0114(8)$ & $0.0116(9)$ \\
C10 & $0.0755(14)$ & $0.0638(13)$ & $0.0380(10)$ & $0.0378(12)$ & $0.0107(9)$ & $0.0170(9)$ \\
C11 & $0.0579(12)$ & $0.0535(11)$ & $0.0383(9)$ & $0.0285(10)$ & $0.0111(9)$ & $0.0104(9)$ \\
C12 & $0.0595(12)$ & $0.0560(12)$ & $0.0463(11)$ & $0.0227(10)$ & $0.0070(9)$ & $0.0132(9)$ \\
C13 & $0.0754(15)$ & $0.0728(15)$ & $0.0434(11)$ & $0.0228(12)$ & $0.0084(10)$ & $0.0220(10)$ \\
C14 & $0.0760(15)$ & $0.0762(15)$ & $0.0417(11)$ & $0.0293(13)$ & $0.0017(11)$ & $0.0157(11)$ \\
C15 & $0.0628(13)$ & $0.0766(15)$ & $0.0529(12)$ & $0.0210(12)$ & $-0.0034(10)$ & $0.0094(11)$ \\
C16 & $0.0576(13)$ & $0.0731(14)$ & $0.0484(11)$ & $0.0181(11)$ & $0.0083(10)$ & $0.0159(10)$ \\
C17 & $0.0690(14)$ & $0.0802(16)$ & $0.0671(14)$ & $0.0124(13)$ & $-0.0022(11)$ & $0.0239(12)$ \\
C18 & $0.0651(13)$ & $0.0687(14)$ & $0.0419(10)$ & $0.0332(11)$ & $0.0089(9)$ & $0.0163(10)$ \\
N1 & $0.0622(10)$ & $0.0564(10)$ & $0.0363(8)$ & $0.0285(8)$ & $0.0113(7)$ & $0.0140(7)$ \\
N2 & $0.0707(11)$ & $0.0640(10)$ & $0.0326(8)$ & $0.0390(9)$ & $0.0089(7)$ & $0.0153(7)$ \\
O1 & $0.0930(11)$ & $0.0775(10)$ & $0.0454(7)$ & $0.0555(9)$ & $0.0183(7)$ & $0.0194(7)$ \\
& & & & & &
\end{tabular}


Geometric parameters $\left(\AA,{ }^{\circ}\right)$

\begin{tabular}{|c|c|c|c|}
\hline $\mathrm{C} 1-\mathrm{N} 1$ & $1.314(2)$ & $\mathrm{C} 10-\mathrm{N} 2$ & $1.334(2)$ \\
\hline $\mathrm{C} 1-\mathrm{N} 2$ & $1.406(2)$ & $\mathrm{C} 10-\mathrm{H} 10$ & 0.9300 \\
\hline $\mathrm{C} 1-\mathrm{C} 2$ & $1.430(2)$ & $\mathrm{C} 11-\mathrm{C} 12$ & $1.390(3)$ \\
\hline $\mathrm{C} 2-\mathrm{C} 18$ & $1.412(2)$ & $\mathrm{C} 11-\mathrm{C} 16$ & $1.393(3)$ \\
\hline $\mathrm{C} 2-\mathrm{C} 7$ & $1.412(2)$ & $\mathrm{C} 12-\mathrm{C} 13$ & $1.393(3)$ \\
\hline $\mathrm{C} 4-\mathrm{C} 18$ & $1.365(3)$ & $\mathrm{C} 12-\mathrm{C} 17$ & $1.500(3)$ \\
\hline $\mathrm{C} 4-\mathrm{C} 5$ & $1.395(3)$ & $\mathrm{C} 13-\mathrm{C} 14$ & $1.367(3)$ \\
\hline $\mathrm{C} 4-\mathrm{H} 4$ & 0.9300 & $\mathrm{C} 13-\mathrm{H} 13$ & 0.9300 \\
\hline $\mathrm{C} 5-\mathrm{C} 6$ & $1.354(3)$ & $\mathrm{C} 14-\mathrm{C} 15$ & $1.368(3)$ \\
\hline $\mathrm{C} 5-\mathrm{H} 5$ & 0.9300 & $\mathrm{C} 14-\mathrm{H} 14$ & 0.9300 \\
\hline $\mathrm{C} 6-\mathrm{C} 7$ & $1.416(3)$ & $\mathrm{C} 15-\mathrm{C} 16$ & $1.388(3)$ \\
\hline $\mathrm{C} 6-\mathrm{H} 6$ & 0.9300 & $\mathrm{C} 15-\mathrm{H} 15$ & 0.9300 \\
\hline $\mathrm{C} 7-\mathrm{C} 8$ & $1.413(2)$ & $\mathrm{C} 16-\mathrm{H} 16$ & 0.9300 \\
\hline $\mathrm{C} 8-\mathrm{C} 9$ & $1.358(3)$ & C17-H17A & 0.9600 \\
\hline $\mathrm{C} 8-\mathrm{H} 8$ & 0.9300 & C17-H17B & 0.9600 \\
\hline $\mathrm{C} 9-\mathrm{N} 1$ & $1.369(2)$ & $\mathrm{C} 17-\mathrm{H} 17 \mathrm{C}$ & 0.9600 \\
\hline $\mathrm{C} 9-\mathrm{C} 11$ & $1.502(2)$ & C18-H18 & 0.9300 \\
\hline $\mathrm{C} 10-\mathrm{O} 1$ & $1.218(2)$ & $\mathrm{N} 2-\mathrm{H} 2$ & 0.8600 \\
\hline $\mathrm{N} 1-\mathrm{C} 1-\mathrm{N} 2$ & $116.00(15)$ & $\mathrm{C} 16-\mathrm{C} 11-\mathrm{C} 9$ & $118.94(17)$ \\
\hline $\mathrm{N} 1-\mathrm{C} 1-\mathrm{C} 2$ & $124.34(16)$ & $\mathrm{C} 11-\mathrm{C} 12-\mathrm{C} 13$ & $118.20(19)$ \\
\hline $\mathrm{N} 2-\mathrm{C} 1-\mathrm{C} 2$ & $119.66(15)$ & $\mathrm{C} 11-\mathrm{C} 12-\mathrm{C} 17$ & $121.62(17)$ \\
\hline $\mathrm{C} 18-\mathrm{C} 2-\mathrm{C} 7$ & $118.91(16)$ & $\mathrm{C} 13-\mathrm{C} 12-\mathrm{C} 17$ & $120.16(19)$ \\
\hline $\mathrm{C} 18-\mathrm{C} 2-\mathrm{C} 1$ & $124.80(16)$ & $\mathrm{C} 14-\mathrm{C} 13-\mathrm{C} 12$ & $121.9(2)$ \\
\hline $\mathrm{C} 7-\mathrm{C} 2-\mathrm{C} 1$ & $116.28(15)$ & $\mathrm{C} 14-\mathrm{C} 13-\mathrm{H} 13$ & 119.0 \\
\hline $\mathrm{C} 18-\mathrm{C} 4-\mathrm{C} 5$ & $120.80(19)$ & $\mathrm{C} 12-\mathrm{C} 13-\mathrm{H} 13$ & 119.0 \\
\hline $\mathrm{C} 18-\mathrm{C} 4-\mathrm{H} 4$ & 119.6 & $\mathrm{C} 13-\mathrm{C} 14-\mathrm{C} 15$ & 120.03 (19) \\
\hline $\mathrm{C} 5-\mathrm{C} 4-\mathrm{H} 4$ & 119.6 & $\mathrm{C} 13-\mathrm{C} 14-\mathrm{H} 14$ & 120.0 \\
\hline $\mathrm{C} 6-\mathrm{C} 5-\mathrm{C} 4$ & $120.51(18)$ & $\mathrm{C} 15-\mathrm{C} 14-\mathrm{H} 14$ & 120.0 \\
\hline $\mathrm{C} 6-\mathrm{C} 5-\mathrm{H} 5$ & 119.7 & $\mathrm{C} 14-\mathrm{C} 15-\mathrm{C} 16$ & $119.5(2)$ \\
\hline $\mathrm{C} 4-\mathrm{C} 5-\mathrm{H} 5$ & 119.7 & $\mathrm{C} 14-\mathrm{C} 15-\mathrm{H} 15$ & 120.2 \\
\hline $\mathrm{C} 5-\mathrm{C} 6-\mathrm{C} 7$ & $120.56(18)$ & $\mathrm{C} 16-\mathrm{C} 15-\mathrm{H} 15$ & 120.2 \\
\hline $\mathrm{C} 5-\mathrm{C} 6-\mathrm{H} 6$ & 119.7 & $\mathrm{C} 15-\mathrm{C} 16-\mathrm{C} 11$ & $120.7(2)$ \\
\hline $\mathrm{C} 7-\mathrm{C} 6-\mathrm{H} 6$ & 119.7 & $\mathrm{C} 15-\mathrm{C} 16-\mathrm{H} 16$ & 119.7 \\
\hline $\mathrm{C} 2-\mathrm{C} 7-\mathrm{C} 8$ & $118.35(16)$ & $\mathrm{C} 11-\mathrm{C} 16-\mathrm{H} 16$ & 119.7 \\
\hline $\mathrm{C} 2-\mathrm{C} 7-\mathrm{C} 6$ & $119.00(16)$ & $\mathrm{C} 12-\mathrm{C} 17-\mathrm{H} 17 \mathrm{~A}$ & 109.5 \\
\hline $\mathrm{C} 8-\mathrm{C} 7-\mathrm{C} 6$ & $122.64(17)$ & $\mathrm{C} 12-\mathrm{C} 17-\mathrm{H} 17 \mathrm{~B}$ & 109.5 \\
\hline $\mathrm{C} 9-\mathrm{C} 8-\mathrm{C} 7$ & $120.43(17)$ & $\mathrm{H} 17 \mathrm{~A}-\mathrm{C} 17-\mathrm{H} 17 \mathrm{~B}$ & 109.5 \\
\hline $\mathrm{C} 9-\mathrm{C} 8-\mathrm{H} 8$ & 119.8 & $\mathrm{C} 12-\mathrm{C} 17-\mathrm{H} 17 \mathrm{C}$ & 109.5 \\
\hline $\mathrm{C} 7-\mathrm{C} 8-\mathrm{H} 8$ & 119.8 & $\mathrm{H} 17 \mathrm{~A}-\mathrm{C} 17-\mathrm{H} 17 \mathrm{C}$ & 109.5 \\
\hline $\mathrm{C} 8-\mathrm{C} 9-\mathrm{N} 1$ & $122.13(16)$ & $\mathrm{H} 17 \mathrm{~B}-\mathrm{C} 17-\mathrm{H} 17 \mathrm{C}$ & 109.5 \\
\hline $\mathrm{C} 8-\mathrm{C} 9-\mathrm{C} 11$ & $122.85(16)$ & $\mathrm{C} 4-\mathrm{C} 18-\mathrm{C} 2$ & $120.22(18)$ \\
\hline $\mathrm{N} 1-\mathrm{C} 9-\mathrm{C} 11$ & $115.00(15)$ & $\mathrm{C} 4-\mathrm{C} 18-\mathrm{H} 18$ & 119.9 \\
\hline $\mathrm{O} 1-\mathrm{C} 10-\mathrm{N} 2$ & $124.36(17)$ & $\mathrm{C} 2-\mathrm{C} 18-\mathrm{H} 18$ & 119.9 \\
\hline $\mathrm{O} 1-\mathrm{C} 10-\mathrm{H} 10$ & 117.8 & $\mathrm{C} 1-\mathrm{N} 1-\mathrm{C} 9$ & $118.43(15)$ \\
\hline $\mathrm{N} 2-\mathrm{C} 10-\mathrm{H} 10$ & 117.8 & $\mathrm{C} 10-\mathrm{N} 2-\mathrm{C} 1$ & $124.96(15)$ \\
\hline
\end{tabular}




$\begin{array}{llll}\mathrm{C} 12-\mathrm{C} 11-\mathrm{C} 16 & 119.63(17) & \mathrm{C} 10-\mathrm{N} 2-\mathrm{H} 2 & 117.5 \\ \mathrm{C} 12-\mathrm{C} 11-\mathrm{C} 9 & 121.41(17) & \mathrm{C} 1-\mathrm{N} 2-\mathrm{H} 2 & 117.5 \\ \mathrm{~N} 1-\mathrm{C} 1-\mathrm{C} 2-\mathrm{C} 18 & 178.11(18) & \mathrm{C} 9-\mathrm{C} 11-\mathrm{C} 12-\mathrm{C} 13 & 176.52(17) \\ \mathrm{N} 2-\mathrm{C} 1-\mathrm{C} 2-\mathrm{C} 18 & -1.4(3) & \mathrm{C} 16-\mathrm{C} 11-\mathrm{C} 12-\mathrm{C} 17 & 179.84(18) \\ \mathrm{N} 1-\mathrm{C} 1-\mathrm{C} 2-\mathrm{C} 7 & -1.8(3) & \mathrm{C} 9-\mathrm{C} 11-\mathrm{C} 12-\mathrm{C} 17 & -1.9(3) \\ \mathrm{N} 2-\mathrm{C} 1-\mathrm{C} 2-\mathrm{C} 7 & 178.68(16) & \mathrm{C} 11-\mathrm{C} 12-\mathrm{C} 13-\mathrm{C} 14 & 1.1(3) \\ \mathrm{C} 18-\mathrm{C} 4-\mathrm{C} 5-\mathrm{C} 6 & -0.4(3) & \mathrm{C} 17-\mathrm{C} 12-\mathrm{C} 13-\mathrm{C} 14 & 179.6(2) \\ \mathrm{C} 4-\mathrm{C} 5-\mathrm{C} 6-\mathrm{C} 7 & 0.6(3) & \mathrm{C} 12-\mathrm{C} 13-\mathrm{C} 14-\mathrm{C} 15 & 0.6(3) \\ \mathrm{C} 18-\mathrm{C} 2-\mathrm{C} 7-\mathrm{C} 8 & -179.13(18) & \mathrm{C} 13-\mathrm{C} 14-\mathrm{C} 15-\mathrm{C} 16 & -1.7(3) \\ \mathrm{C} 1-\mathrm{C} 2-\mathrm{C} 7-\mathrm{C} 8 & 0.8(3) & \mathrm{C} 14-\mathrm{C} 15-\mathrm{C} 16-\mathrm{C} 11 & 1.0(3) \\ \mathrm{C} 18-\mathrm{C} 2-\mathrm{C} 7-\mathrm{C} 6 & 0.1(3) & \mathrm{C} 12-\mathrm{C} 11-\mathrm{C} 16-\mathrm{C} 15 & 0.7(3) \\ \mathrm{C} 1-\mathrm{C} 2-\mathrm{C} 7-\mathrm{C} 6 & -179.99(17) & \mathrm{C} 9-\mathrm{C} 11-\mathrm{C} 16-\mathrm{C} 15 & -177.58(18) \\ \mathrm{C} 5-\mathrm{C} 6-\mathrm{C} 7-\mathrm{C} 2 & -0.4(3) & \mathrm{C} 5-\mathrm{C} 4-\mathrm{C} 18-\mathrm{C} 2 & 0.0(3) \\ \mathrm{C} 5-\mathrm{C} 6-\mathrm{C} 7-\mathrm{C} 8 & 178.7(2) & \mathrm{C} 7-\mathrm{C} 2-\mathrm{C} 18-\mathrm{C} 4 & 0.1(3) \\ \mathrm{C} 2-\mathrm{C} 7-\mathrm{C} 8-\mathrm{C} 9 & 0.8(3) & \mathrm{C} 1-\mathrm{C} 2-\mathrm{C} 18-\mathrm{C} 4 & -179.81(19) \\ \mathrm{C} 6-\mathrm{C} 7-\mathrm{C} 8-\mathrm{C} 9 & -178.41(19) & \mathrm{N} 2-\mathrm{C} 1-\mathrm{N} 1-\mathrm{C} 9 & -179.35(16) \\ \mathrm{C} 7-\mathrm{C} 8-\mathrm{C} 9-\mathrm{N} 1 & -1.5(3) & \mathrm{C} 2-\mathrm{C} 1-\mathrm{N} 1-\mathrm{C} 9 & 1.1(3) \\ \mathrm{C} 7-\mathrm{C} 8-\mathrm{C} 9-\mathrm{C} 11 & 176.90(18) & \mathrm{C} 8-\mathrm{C} 9-\mathrm{N} 1-\mathrm{C} 1 & 0.6(3) \\ \mathrm{C} 8-\mathrm{C} 9-\mathrm{C} 11-\mathrm{C} 12 & 83.5(3) & \mathrm{C} 11-\mathrm{C} 9-\mathrm{N} 1-\mathrm{C} 1 & -177.95(17) \\ \mathrm{N} 1-\mathrm{C} 9-\mathrm{C} 11-\mathrm{C} 12 & -98.0(2) & \mathrm{O} 1-\mathrm{C} 10-\mathrm{N} 2-\mathrm{C} 1 & -177.35(19) \\ \mathrm{C} 8-\mathrm{C} 9-\mathrm{C} 11-\mathrm{C} 16 & -98.3(2) & \mathrm{N} 1-\mathrm{C} 1-\mathrm{N} 2-\mathrm{C} 10 & -1.6(3) \\ \mathrm{N} 1-\mathrm{C} 9-\mathrm{C} 11-\mathrm{C} 16 & 80.3(2) & \mathrm{C} 2-\mathrm{C} 1-\mathrm{N} 2-\mathrm{C} 10 & 177.92(17) \\ \mathrm{C} 16-\mathrm{C} 11-\mathrm{C} 12-\mathrm{C} 13 & -1.7(3) & & \\ & & & \end{array}$

Hydrogen-bond geometry $\left(\AA,{ }^{\circ}\right)$

\begin{tabular}{lllll}
\hline$D-\mathrm{H} \cdots A$ & $D-\mathrm{H}$ & $\mathrm{H} \cdots A$ & $D \cdots A$ & $D-\mathrm{H} \cdots A$ \\
\hline $\mathrm{N} 2-\mathrm{H} 2 \cdots \mathrm{O}^{\mathrm{i}}$ & 0.86 & 2.10 & $2.940(2)$ & 165 \\
\hline
\end{tabular}

Symmetry code: (i) $-x+1,-y+1,-z+1$. 